\title{
Note on Transcription and Pronunciation
}

I used both Nepali and the local dialect of Tamang in my research. I am relatively adept in both but have studied Nepali for many more years. Nepali was my medium for learning Tamang, and I often asked for explanations in Nepali when I did not fully understand what had been said in Tamang. Many Tamang are bilingual, and I moved in and out of Tamang and Nepali while conversing. Tamang ritual orations and certain songs also employ an archaic form of Tamang, which most villagers, including ritual specialists, cannot translate completely word for word; they almost always know, though, the general sense and implications of their words. Nepali words, written in devanāgar $\bar{\imath}$ script, are transcribed here for the most part according to Turner's Comparative and Etymological Dictionary of the Nepali Language ([193I] 1965). I have made several exceptions to Turner's system to facilitate reading for nonspecialists. Turner's "c," "ch," "ín," and "'s" are rendered, respectively, as "ch," "chh," "ng," and "sh." The names of well-known ethnic and caste groups of Nepal and other well-known Nepali terms appear as they are commonly written without diacritical marks.

Tamang is an unwritten Tibeto-Burman language and has several significant tones. It varies from village to village and there is no standard. When I first began to live in Tamdungsa, I developed a roughand-ready system for transcribing the local dialect into Devanagari. Thus, I have transcribed Tamang words, with a few exceptions, as I have transcribed Nepali words. What sounds approximately like "shy" to English speakers is rendered by Tamang in Devanagari as "sy." I have opted here to write "shy" for the Devanagari "sy" as in "shyingo." The Tamang phonemes "ts," "tsh," and "ds" are not recognized in 
Devanagari, and I have transcribed them as in the beginning of this sentence. The only Tamang pitch or tone that is marked in this book is the breathy, which is indicated by an " $h$ " after a consonant that does not have an aspirated form or after a vowel that succeeds a consonant that does have an aspirated form. The transcriptions are somewhat different from and more accurate than what I have used before (Holmberg 1980, 1983, I984). I make no claims to linguistic precision, and many significant phonetic distinctions are lost in this system of transcription. For those who require more accurate transcriptions of western Tamang, many if not all the terms used here appear with greater precision in Höfer (I98I).

As transcribed here, both singular and plural forms of Tamang words are rendered in singular form. The only exceptions to this practice are the recurring terms for ritual practitioners-lama, bombo, and lambu - which are made plural by adding an "s." Tibetan terms are transcribed according to the sources from which they were drawn. If no source is apparent, I have tried to be consistent with the Wylie system (Wylie 1959) as used in Tucci (r980). Sanskrit terms are written without diacritical marks and in approximation of English pronunciation. Non-English terms appear in italics and with diacritical marks only in the first instance of their use or when they are reintroduced after many pages. A glossary of the commonly used non-English terms appears at the end of the text. All translations are mine unless otherwise indicated.

Many readers, especially those who read this text from perspectives steeped in Tibetology, greater Nepal studies, Indology, or Asian studies, will be vexed by my use of Tamang terms instead of more standardized Tibetan, Nepali, or Sanskrit terms. For example, I use the local form guru rhimborochhe for a Buddhist hero instead of the Tibetan $G u$ $r u$ Rin po che or Sanskrit padmasambhava. This Tamang-centric practice conforms with the overall orientation of this text, which attempts to reconstruct cultural forms in Nepal from Tamang and Nepalese perspectives, and to understand Nepalese culture not simply as a derivation of greater Indic or Tibetan formations. 
OR D E R I N P A R A D O X 
